\title{
Vibration of Single-Walled Carbon Nanotubes by Using Nonlocal Theory
}

\author{
Jozef Bocko*, Pavol Lengvarský \\ Department of Applied Mechanics and Mechanical Engineering, Technical University of Košice, Faculty of Mechanical Engineering, \\ Košice, Slovakia \\ *Corresponding author: jozef.bocko@tuke.sk
}

Received September 02, 2014; Revised September 17, 2014; Accepted October 06, 2014

\begin{abstract}
The free bending vibration of single-walled carbon nanotubes (SWCNTs) is investigated in the present paper. A continuum approach based on nonlocal theory of beam bending is used for natural frequency computation. Analytical solutions of frequency equations are given for four types boundary conditions: clamped-free (C-F), simply-simply supported (S-S), clamped-simply supported (C-S) and clamped-clamped (C-C). The graphical representations of numerical results are shown for the first case of boundary conditions clamped end - free end.
\end{abstract}

Keywords: single-walled carbon nanotube, vibration, beam, nonlocal theory, free vibration

Cite This Article: Jozef Bocko, and Pavol Lengvarský, "Vibration of Single-Walled Carbon Nanotubes by Using Nonlocal Theory.” American Journal of Mechanical Engineering, vol. 2, no. 7 (2014): 195-198. doi: 10.12691/ajme-2-7-5.

\section{Introduction}

Carbon nanotubes and underlying research are connected with Iijima and his famous paper [7]. Carbon nanotubes are closed graphene sheets of cylindrical shape with interesting mechanical properties. Especially, high tensile strength and stiffness in relation to very small weight attracts attention of engineers and researches. However, not only mechanical properties of nanotubes are interesting. Intensive research is also oriented to the application in electrical and chemical engineering, as well as in biological sciences. There have been some researches on the vibration problems of SWCNTs through experiments, but at the same time theoretical treatment is used for description of measured data. Three basic methods of investigation are used to accomplish simulation of SWCNTs: molecular dynamic simulations, atomistic-based modeling and the continuum approach. The last one is used here for description of bending vibration and accordingly it can serve as a mean for indirect specification of carbon nanotube properties. The continuum beam relations used in the paper are based on the theory of nonlocal elasticity published by Eringen [4]. In contrast to the classical elasticity theory Eringen takes into account the scale effect and its influence to stress at the reference point of a body. Accordingly, the stress at a point is a function of strain field at every point of body. Review of nonlocal elastic models of carbon nanotubes is given in [1] and nonlocal theory based on Euler beams is described in [9]. The interesting results of SWCNT research can be found in literature [1-6,8,10,13,15].

\section{Bending Vibrations of Carbon Nanotubes}

\subsection{Theoretical Background}

The classical elasticity theory uses assumption of local action of physical quantities. It means that the stress at a given point correspondence to the strains in its intimate neighborhoods. In the nonlocal theory the stress state at the point of question is influenced by strains in all points of the investigated body [4]. In accordance with this assumption, the behavior of homogeneous isotropic beam can be described by the following equation

$$
\sigma_{x x}-\mu \frac{\partial^{2} \sigma_{x x}}{\partial x^{2}}=E \varepsilon_{x x},
$$

where $\sigma_{x X}, \varepsilon_{x X}, E$ are normal stress, normal strain and Young's modulus, respectively. The scaling factor $\mu$ at the second term on the left side of equation reflects the effect of small length scale. This factor can be expressed by equation $\mu=e_{0}^{2} l_{i}^{2}$, where $e_{0}$ is the material constant and $l_{i}$ is an internal characteristic length. In classical theory, parameter $\mu$ is used for modeling of bending, buckling and free vibration problems of beams [12]. The Euler assumptions for free vibration of beam together with the nonlocal theory lead to equation [9]

$$
m(x) \mu \frac{\partial^{2} u}{\partial t^{2}}+\frac{\partial^{2}}{\partial x^{2}}\left[E I(x) \frac{\partial^{2} u}{\partial x^{2}}\right]=0 .
$$

Here, $m, I$ and $u$ are the mass per unit length, the moment of inertia of cross-section area and the transverse displacement of a nanotube, respectively. Fourier method of separation of variables leads to the system of two ordinary differential equations. One equation is related to time variable and the second one is connected with spatial variable $x$, describing axial position of point in question. 
Assumption that nanotube has a constant cross-section area leads to differential equation

$$
\varphi^{I V}(x)-\beta^{4} \varphi(x)=0,
$$

where $\varphi(x)$ is an eigenshape (mode shape) and for parameter $\beta$ we have equation

$$
\beta^{4}=\frac{\omega^{2} \mu m}{E I} .
$$

The quantity $\omega$ in equation (4) represents an angular frequency of free vibration of nanotube and by using $\beta$ the equation (3) leads to the solution

$$
\begin{aligned}
\varphi(x)= & C_{1} \sin (\beta x)+C_{2} \cos (\beta x)+ \\
& C_{3} \sinh (\beta x)+C_{4} \cosh (\beta x) .
\end{aligned}
$$

Here, $C_{1}, C_{2}, C_{3}, C_{4}$ are the constants depending on boundary conditions applied to the ends of investigated beam.

\subsection{Solutions that Correspond to Different Boundary Conditions}

Free vibrations of the armchair type of carbon nanotubes with boundary conditions: clamped-free (C-F), simply-simply supported (S-S), clamped-simply supported (C-S) and clamped-clamped (C-C) are solved in the paper. The length of carbon nanotube is $L$ and the lattice translation indices $(n, m)$ for armchair nanotubes can be written as $(n, n)$. Schema of graphene sheet and carbon nanotube is given in Figure 1.

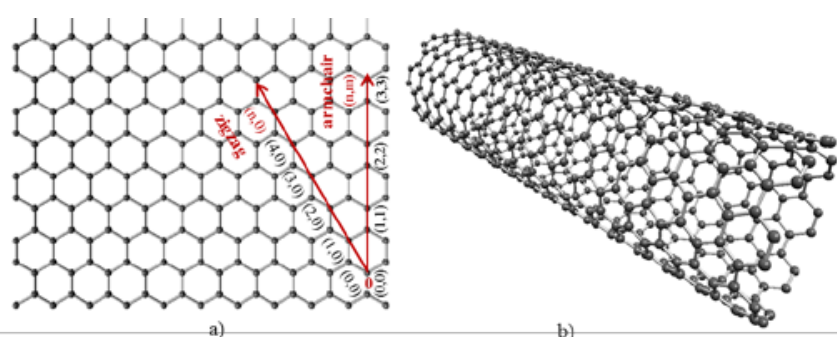

Figure 1. Hexagonal lattice a) sheet b) single-walled nanotube

The frequency equations for above-mentioned boundary conditions are

$$
\begin{gathered}
1+\cos (\beta L) \cosh (\beta L)=0, \\
\sin (\beta L) \sinh (\beta L)=0,
\end{gathered}
$$

(C-S): $\sinh (\beta L) \cos (\beta L)-\cosh (\beta L) \sin (\beta L)=0$,

(C-C):

$$
\begin{aligned}
& \cos ^{2}(\beta L)+\cosh ^{2}(\beta L)- \\
& 2[\cos (\beta L) \cosh (\beta L)]=0 .
\end{aligned}
$$

The continuum approach leads to the fact that the frequency equations for given boundary conditions have infinite number of solutions $(\beta L)$. The values of $(\beta L)$ for the first 15 eigenfrequencies (natural frequencies) were computed numerically in Matlab and they are given in Table 1.

The angular frequencies $\omega$ are computed according to equation (4) and frequencies $f$ from relation $f=\omega / 2 \pi$.
In case of nanotubes there is uncertainty in definition of thickness of the nanotube shell. However, in the literature are given some relations that can be used in (4). Here, we apply relations from [14]

$$
m=\rho A=2.4 \times 10^{-24} D \quad[\mathrm{~kg} / \mathrm{nm}],
$$

Table 1. Solutions of Frequency Equations

\begin{tabular}{|c|c|c|c|c|}
\hline \multirow{2}{*}{$\begin{array}{c}\text { Eigenfre-quency } \\
\text { No. }\end{array}$} & \multicolumn{4}{|c|}{ Value of $(\beta L)$ for boundary conditions } \\
\cline { 2 - 5 } & C-C & C-S & C-F & S-S \\
\hline 1. & 4.7300407 & 3.9266023 & 1.8751040 & 3.1415926 \\
\hline 2. & 7.8532046 & 7.0685827 & 4.6940911 & 6.2831853 \\
\hline 3. & 10.995607 & 10.210176 & 7.8547574 & 9.4247779 \\
\hline 4. & 14.137165 & 13.351768 & 10.995540 & 12.566370 \\
\hline 5. & 17.278759 & 16.493361 & 14.137168 & 15.707963 \\
\hline 6. & 20.420352 & 19.634954 & 17.278759 & 18.849555 \\
\hline 7. & 23.561944 & 22.776546 & 20.420352 & 21.991148 \\
\hline 8. & 26.703537 & 25.918139 & 23.561944 & 25.132741 \\
\hline 9. & 29.845130 & 29.059732 & 26.703537 & 28.274333 \\
\hline 10. & 32.986722 & 32.201324 & 29.845130 & 31.415926 \\
\hline 11. & 36.128315 & 35.342917 & 32.986722 & 34.557519 \\
\hline 12. & 39.269908 & 38.484510 & 36.128315 & 37.699111 \\
\hline 13. & 42.411500 & 41.626102 & 39.269908 & 40.840704 \\
\hline 14. & 45.553093 & 44.767695 & 42.411500 & 43.982297 \\
\hline 15. & 48.694686 & 47.909287 & 45.553093 & 47.123889 \\
\hline
\end{tabular}

$E I=428.48 D^{2}-397.08 D+109.24\left[\mathrm{~kg} \mathrm{~nm}^{3} / \mathrm{s}^{2}\right]$,

where $D$ is a diameter of nanotube. The nanotube diameter can be computed from translation indices $(n, m)$ by relation

$$
D=2 R=a_{0} \sqrt{3\left(m^{2}+n^{2}+m n\right)} / \pi,
$$

where $a_{0}=1.42 \AA$ is a carbon-carbon bond length and for armchair nanotube we have $m=n$. Due to the fact that huge number of possible parameter combination can occur, the results are given only for the first four eigenfrequencies for diameters $D=1,356 \mathrm{~nm}$ and $D=2,034 \mathrm{~nm}$. The graphs on Figure 2 to Figure 11 show dependencies of eigenfrequencies on the length $L$ of carbon nanotube with boundary conditions of type C-F. The nonlocal parameter is $0.01,0.04,0.1,0.3$ and 0.4 , respectively.

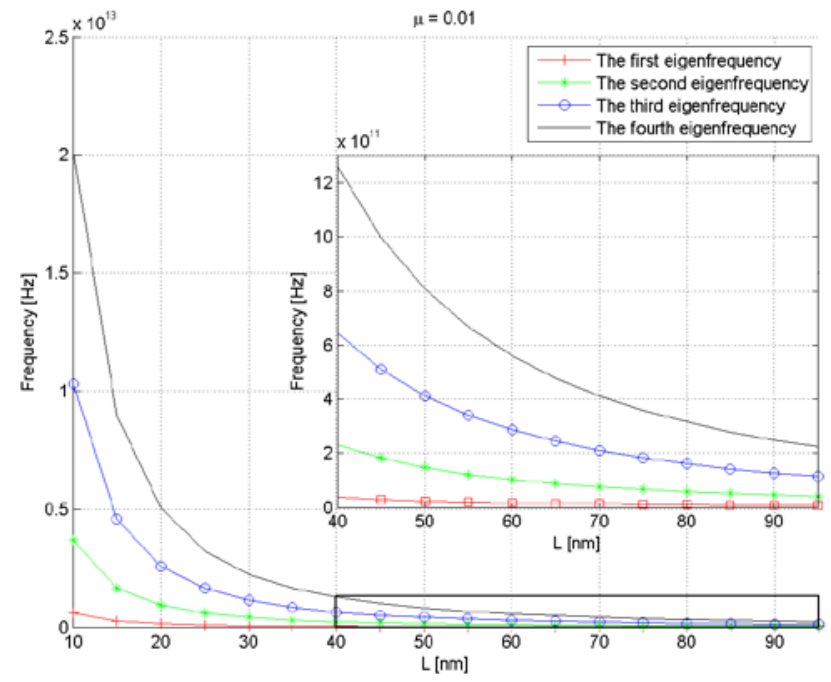

Figure 2. The first four eigenfrequencies for diameter $D=1,356 \mathrm{~nm}$ and nonlocal parameter $\mu=0.01$ 


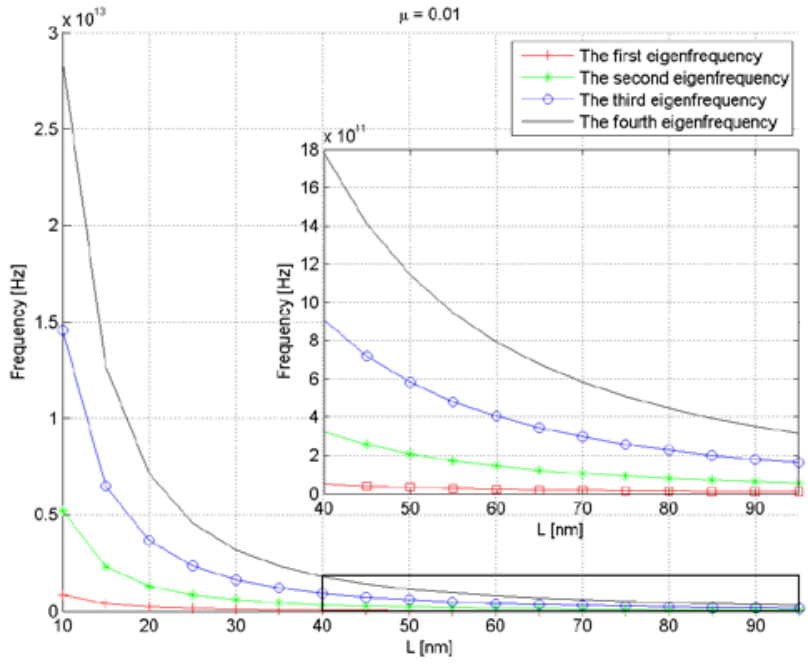

Figure 3. The first four eigenfrequencies for diameter $\mathrm{D}=2,034 \mathrm{~nm}$ and nonlocal parameter $\mu=0.01$

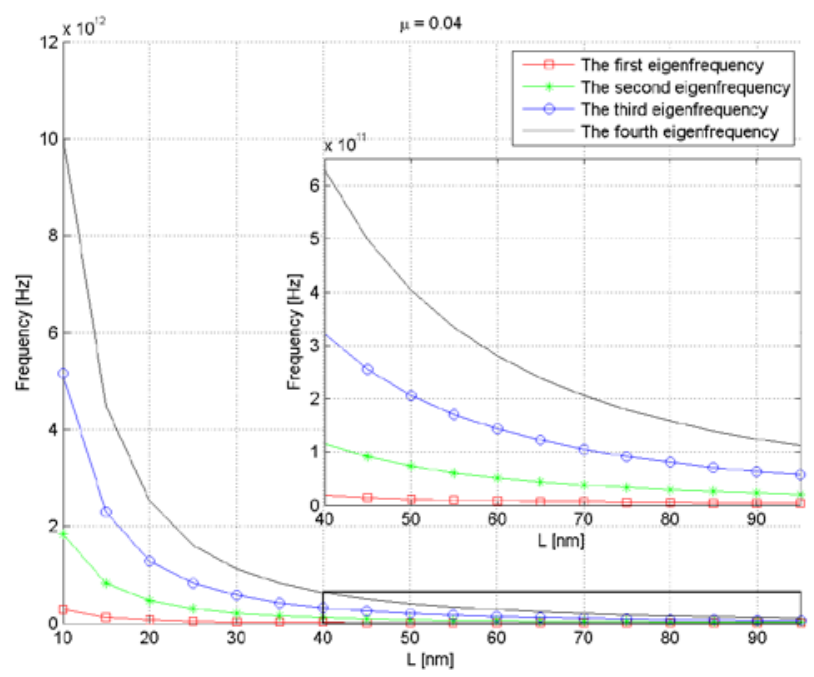

Figure 4. The first four eigenfrequencies for diameter $\mathrm{D}=1,356 \mathrm{~nm}$ and nonlocal parameter $\mu=0.04$

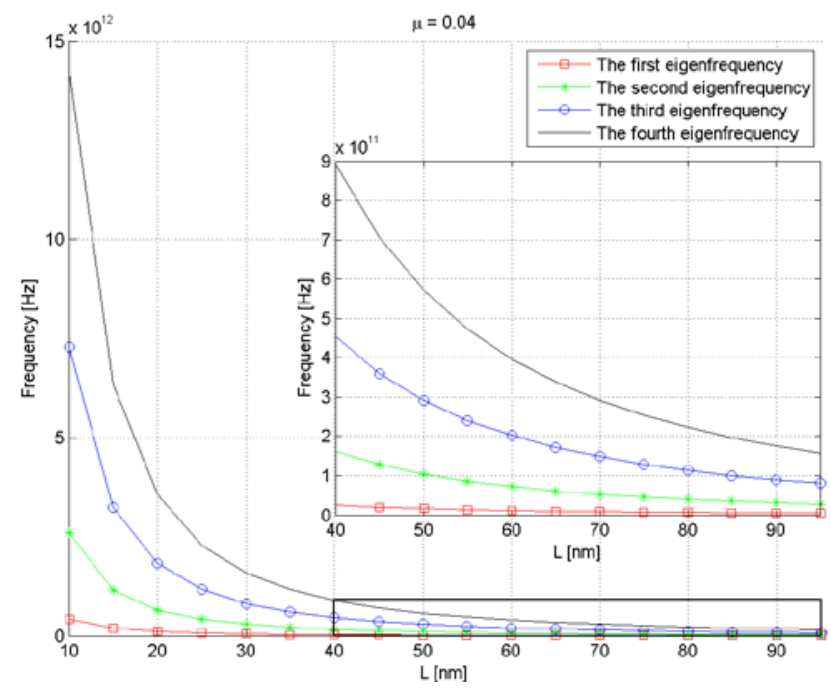

Figure 5. The first four eigenfrequencies for diameter $\mathrm{D}=2,034 \mathrm{~nm}$ and nonlocal parameter $\mu=0.04$

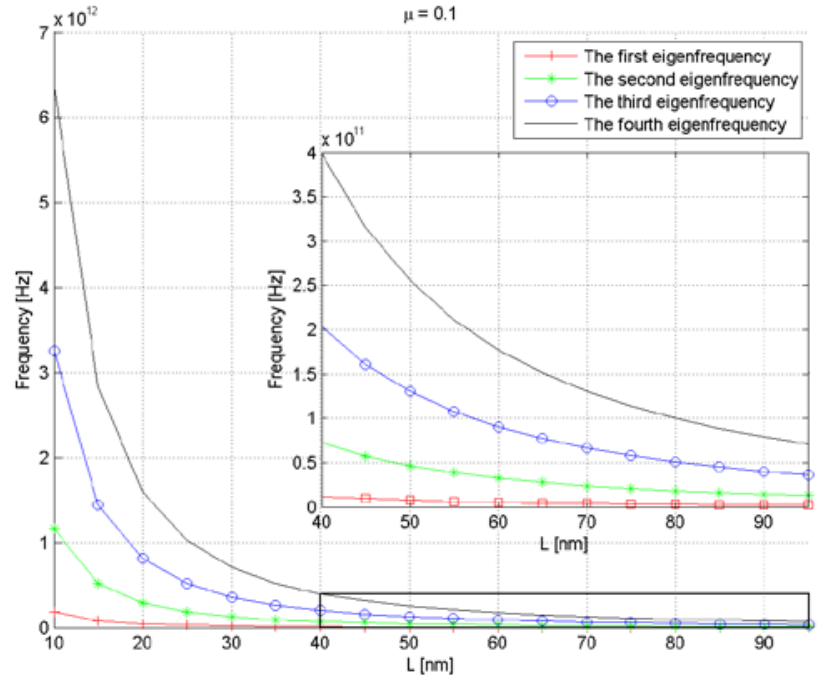

Figure 6. The first four eigenfrequencies for diameter $D=1,356 \mathrm{~nm}$ and nonlocal parameter $\mu=0.1$

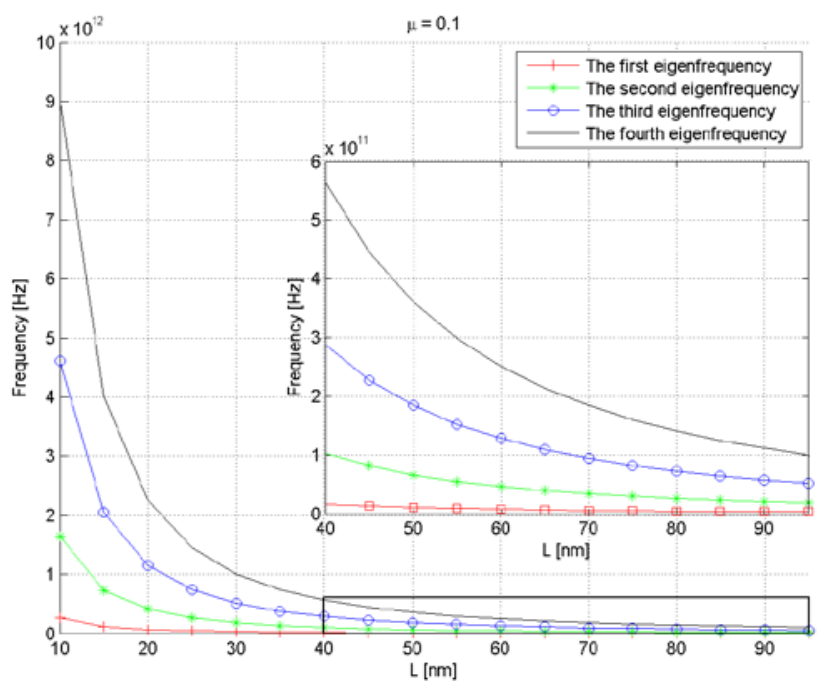

Figure 7. The first four eigenfrequencies for diameter $D=2,034 \mathrm{~nm}$ and nonlocal parameter $\mu=0.1$

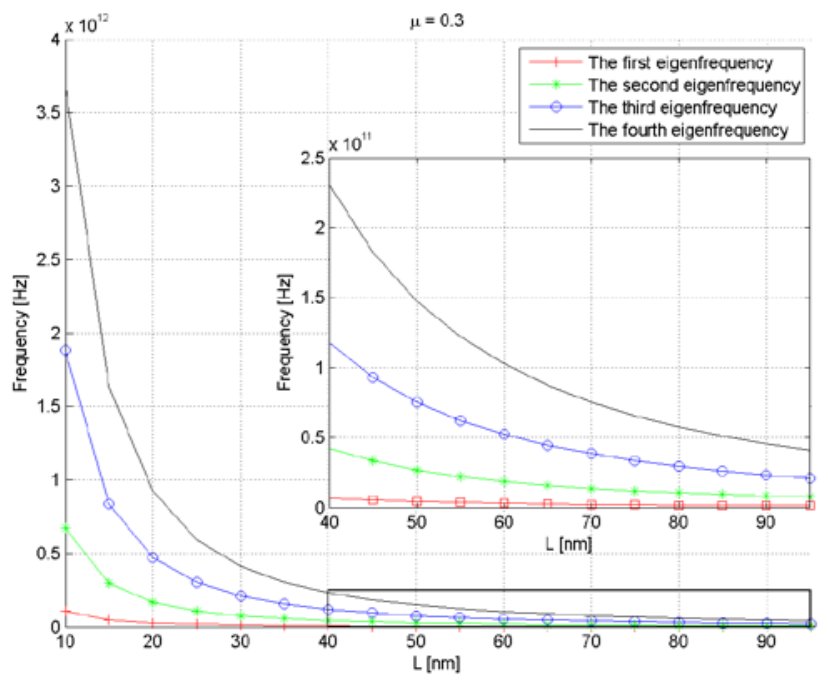

Figure 8. The first four eigenfrequencies for diameter $D=1,356 \mathrm{~nm}$ and nonlocal parameter $\mu=0.3$ 


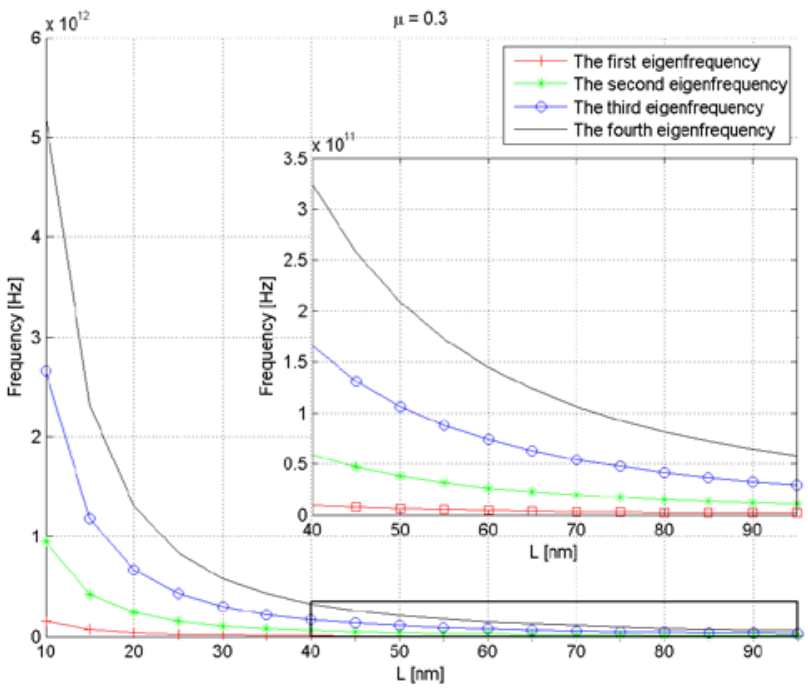

Figure 9. The first four eigenfrequencies for diameter $D=2,034$ and nonlocal parameter $\mu=0.3$

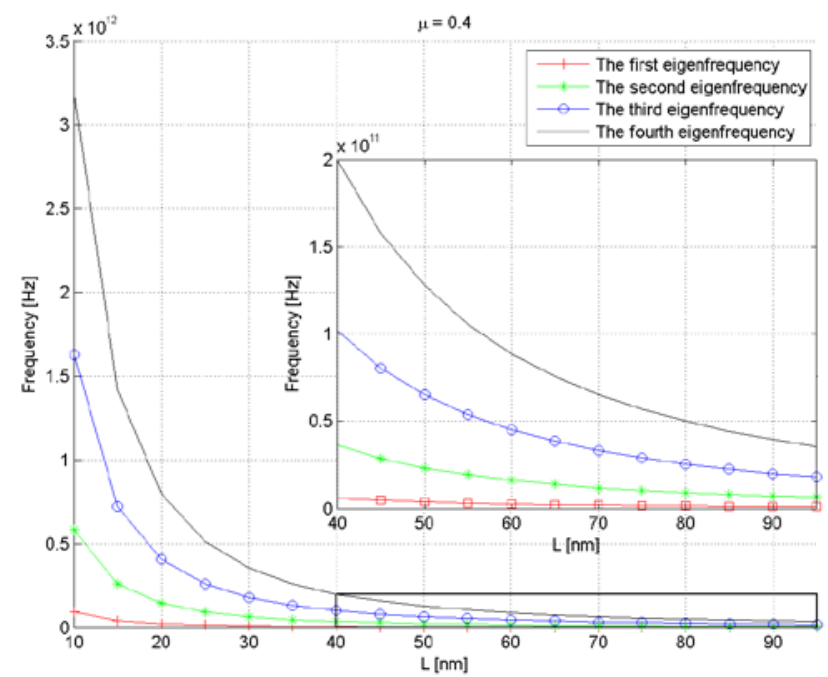

Figure 10. The first four eigenfrequencies for diameter $D=1,356$ and nonlocal parameter $\mu=0.4$

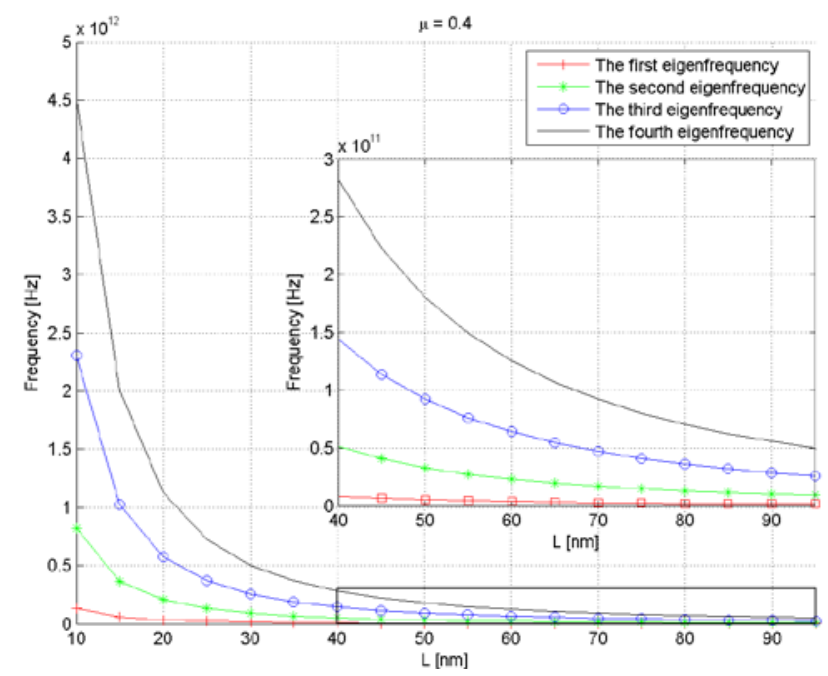

Figure 11. The first four eigenfrequencies for diameter $D=2,034$ and nonlocal parameter $\mu=0.4$

\section{Conclusions}

The paper deals with the free bending vibration of armchair single-walled carbon nanotubes. The study is based on the nonlocal beam theory, where the stress state at the investigated point is influenced by strains in all points of the investigated body. The solutions of frequency equations are given for four types of boundary conditions in the table and specific results for cantilever beam are given in several graphs. The eigenfrequencies are given there for two different diameters of nanotube, several nonlocal parameters and continuously changed length.

\section{Acknowledgement}

This research was undertaken thanks to funding from Slovak Grant Agency VEGA 1/1205/12 and KEGA 054TUKE-4/2014.

\section{References}

[1] Eringen, A.C., Nonlocal continuum field theories, Springer-Verlag, 2002.

[2] Grupta, S.S., Barta, R.C., "Continuum structures equivalent in normal mode vibrations to single-waled carbon nanotubes”, Comp. Mater. Sci., 43, 715-723, 2008.

[3] Harik, V.M., "Mechanics of carbon nanotubes: applicability of the continuum-beam models”, Comp. Mater. Sci., 24, 328-342, 2002.

[4] Iijima, S., "Helical microtubules of graphitic carbon", Nature, 345 , 56-58, 1991.

[5] Li, C.Y., Chou, T., "A structural mechanics approach for the analysis of carbon nanotubes", Int. J. of Solids and Struct., 40, 2487-2499, 2003.

[6] Lu, J., Chen, H., Lu, P., Zhang, P., "Research of natural frequency of single-walled carbon nanotube”, Chinese J. of Chem. Phys., 20, 525-530, 2007.

[7] Lu, P., Lee, H.P., Lu, C., Zhang, P.Q., “Application of nonlocal beam model for carbon nanotubes”, Int. J. of Solids and Struct., 44 5289-5300, 2007.

[8] Muc, A., Banas, A., Chwal, M., "Free vibrations of carbon nanotubes with defects”, Mech. and Mechan. Eng., 17, 157-166, 2013.

[9] Reddy, J.N., Pang, S.D., "Nonlocal continuum theories of beams for the analysis of carbon nanotubes", J. of Appl. Phys., 103, 023511-1-023511-16, 2008.

[10] Sakhaee-Pour, A., Ahmadian, M.T., Vafai, A. "Vibrational analysis of single-walled carbon nanotubes using beam element", Thin-walled struct., 47, 646-652, 2009.

[11] Swain, A., Roy, T., Nanda, B.K., "Vibration behaviour of single walled carbon nanotube using finite element”, Int. J. Theor. and Appl. Res. in Mech. Eng., 2, 129-133, 2013.

[12] Thongyothee, C., Chucheepsakul, S., "Finite element modeling of van der Waals interaction for elastic stability of multi-walled carbon nanotubes”, Adv. Mater. Res., 55-57, 525-528, 2008.

[13] Thongyothee, C., Chucheepsakul, S., Li, T., "Nonlocal elasticity theory for free vibration of single-walled carbon nanotubes", Adv. Mater. Res., 747, 257-260, 2013.

[14] Tserpes, K.I., Papanikos, P., "Finite element modeling of singlewalled carbon nanotubes”, Comp. Part B: Eng., 36, 468-477, 2005.

[15] Wang, Q., Liew, K.M., "Application of nonlocal continuum mechanics to static analysis of micro-and nano-structures", Physics Letters A, 363, 236-242, 2007. 